\title{
Editorial
}

Psychotherapy
and Psychosomatics

Psychother Psychosom 2017;86:323-331

Received: July 24, 2017

DOI: $10.1159 / 000479820$

Accepted: July 25, 2017

Published online: November 3, 2017

\section{Clinical Factor 2016}

\author{
Richard Balon
}

Department of Psychiatry and Behavioral Neurosciences and Department of Anesthesiology, Wayne State

University School of Medicine, Detroit, MI, USA

As in the previous 6 years I am presenting my subjective selection, this time of 13 (primary) articles - actually their summaries - published in the previous year (2016) that I believe may provide some information to help the reader improve his/her clinical practice. As I noted before, the idea was originally proposed by Giovanni Fava [1] with the goal of selecting papers that I would subjectively perceive as having a high "clinical factor" - the degree and extent to which a journal article provides information to the clinician that may improve his/her practice. And again, as I wrote before about the selected articles, "many may find them useful, some will find them less useful or even useless. I am certainly biased, as is anybody else. I also cannot review all psychiatric journals - I have focused only on most of the major ones. If there is no article selected from a certain major psychiatric journal, it does not necessarily mean that I have not reviewed it or skimmed through that journal. It may mean that I did not find, in my opinion, anything clinically very interesting" [2]. I view myself first and foremost as a clinician (though academically oriented) and educator, and I am, as most clinicians are, looking for a way to help patients and for guidance in the treatment of my patients, and thus for new, innovative, or newly validated treatments. I am sure I missed a number of valuable articles, but as noted, this is a subjective selection.

\section{KARGER}

๑ 2017 S. Karger AG, Basel

E-Mail karger@karger.com

www.karger.com/pps

\section{Schizophrenia}

As Niemela et al. [3] point out, increasing evidence supports a role for pre- and postnatal insults that affect neurodevelopment in schizophrenia. One such possible insult is maternal smoking. Thus, Niemela et al. [3] examined the relationship between prenatal nicotine exposure (cotinine levels measured by immunoassay) in archived maternal sera and schizophrenia in offspring from a Finnish national birth cohort (Finnish Population Registry) in a nested case-control design. The sample consisted of cases of schizophrenia in offspring $(n=977)$ born in Finland from 1983 to 1998. Subjects were followed until 2009. The cases were matched 1:1 to controls on date of birth, sex, and residence in Finland at the time of case diagnosis. The covariates included sex, maternal age, paternal age, socioeconomic status (maternal education), province of birth, municipality of birth, parental history of schizophrenia and other psychiatric disorders, previous births, weight for gestational age, maternal C-reactive protein level, and gestational week of the maternal blood draw. In the unadjusted analysis with cotinine as a continuous variable and taking the presence of cotinine levels below the level of detection into account, there was a significant association between increased log-transformed maternal cotinine and odds of schizophrenia (odds ratio $=2.69,95 \% \mathrm{CI}=1.54-$

Richard Balon, MD

Departments of Psychiatry and Behavioral Neurosciences and Anesthesiology Tolan Park Building, Third Floor, 3901 Chrysler Service Dr. Detroit, MI 48201 (USA)

E-Mail rbalon@wayne.edu 
$4.69, p<0.0001)$. In a model including maternal age, province of birth, and any paternal psychiatric disorder as covariates, the odds ratio was 3.41 (95\% CI $=1.86-6.24, p<$ $0.0001)$. Importantly, there was no clear evidence that weight for gestational age mediated the association. Categorically defined heavy maternal nicotine exposure was related to a $38 \%$ increased odds of schizophrenia. The authors pointed out several limitations to their study, such as lack of data on maternal use of alcohol (there is an association between alcohol use and smoking), the fact that nicotine is just one of many toxic substances in tobacco, the relatively young age of their cohort (median age 23.5 years), lack of data on offspring smoking, lack of data on parental second-hand smoking, and multiple comparisons used. Nevertheless, this is an important finding - as Laursen and McGrath [4] pointed out in their accompanying editorial, we do not have many modifiable candidate risk factors for schizophrenia, so the identification of smoking as a potential modifiable risk factor is quite important. They [4] also wrote that though this finding is not "beyond reasonable doubt," it is clear that smoking is bad for one's health and pregnant women who smoke put their children's health at risk, too. (The Niemela study was supported by NIMH grants and a grant from the National Institute of General Medical Sciences. Dr. Niemela received honoraria and travel funds from Janssen and Lundbeck, and honoraria from Lilly. Dr. Laursen is supported by Lundbeck Foundation Initiative Psychiatric Research and Mental Health in Primary Care; Dr. McGrath is supported by a grant from the John Cade fellowship from the National Health and Medical Research Council.)

Adolescents engaging in antisocial behavior are at an elevated risk of schizophrenia and also for substance misuse and offending, which complicates their compliance with treatment. The relationship between various problem behaviors in adolescence and schizophrenia is a complex one - individuals who develop schizophrenia have an increased frequency of conduct disorder in childhood/ adolescence, large proportions of first-episode patients have a history of criminality and substance misuse, the incidence of psychotic disorder in young delinquents is higher than in the general population, and heavy use of cannabis during adolescence is associated with an increased risk of schizophrenia. Considering all these factors, Hodgins et al. [5] studied adolescents in a large urban center in Sweden to determine whether adolescents seeking treatment for substance misuse were at a higher risk of developing schizophrenia compared to age- and sex-matched general population peers, whether this risk has changed since 1960, whether cannabis misuse in ado- lescence predicted the development of schizophrenia, and whether adolescent substance misuse predicted substance use disorders, physical disorders, criminal convictions, poverty and death in those who developed schizophrenia. They obtained 3 population samples from the only clinic for adolescent substance misuse in Stockholm: 1,992 individuals (1,660 males, 332 females) treated from 1968 to $1971 ; 1,576$ individuals $(1,010$ males and $566 \mathrm{fe}$ males) treated from 1980 to 1984 (matched samples were obtained for both samples), and 180 individuals (81 males, 99 females) followed at the clinic between 2000 and 2009. The first sample was followed until the age of 50 years, the second until the age of 35 years, and the last sample was followed at 6,12 , and 60 months after the admission to the clinic. The prevalence of schizophrenia was higher in the first and second sample when compared to matched samples, the increase was approximately 4-fold for males and 7- to 8-fold for females. In the third sample, $11 \%$ of males and $14 \%$ of females had a diagnosis of schizophrenia, as compared with $1.0 \%$ of the males and $0.6 \%$ of the females of similar age in Stockholm at the age of 21.7 years (the initial evaluation was done at the age of 17.3 years). There was no increased risk over the time between the sample treated during 1968 and 1971 and the sample treated from 1980 through 1984. Among males but not females in both first and second samples, a greater proportion of those who did as compared to those who did not use cannabis developed schizophrenia. Interestingly, among both males and females in the first and second samples and among females in the third sample, a greater proportion of those who developed schizophrenia used stimulants. The limitations included small size of the third sample, lack of information about the matched population samples beyond the fact that they were not treated for substance misuse, and the fact that the rates of schizophrenia may have been underestimated, as they were based only on hospitalized cases. Nevertheless, it seems that a disproportionate number of adolescents misusing substances develop schizophrenia later on. We need to pay more attention to early detection of those cases and to possible prevention. (This study was supported by grants from Stockholm City Council. The authors declared no conflict of interest.)

\section{Psychotherapy}

Various psychotherapies have been found effective in the treatment of anxiety and mood disorders in adolescents. However, many adolescents do not engage in psy- 
chotherapy. Motivational interviewing (MI) has been described as a conversational approach to enhance the person's motivation and commitment to change and has been used as a stand-alone treatment for addictive behaviors and health behavior change. Lately, MI has also been used as a pretreatment intervention with the aim to improve gains from subsequent treatment. Dean et al. [6] examined whether MI would enhance treatment involvement in a group cognitive behavioral therapy (CBT, i.e., gCBT) in adolescents treated for anxiety and mood disorders. Their population sample consisted of 96 adolescents treated at 2 publicly funded outpatient services in Wellington, New Zealand. They were assigned to either 1 session of MI (46 patients) or 1 session of befriending (50 patients), up to $50 \mathrm{~min}$ in duration. Befriending, used as control psychotherapy for clinical trials, is a nonspecific and nondirective intervention focused on a neutral topic. These sessions were followed by 5 gCBT sessions about 120 min each. Participation in gCBT served as the primary outcome measure. Participants in the pretreatment MI attended significantly more gCBT sessions (mean $=4.0$, range $=2.5-5.0)$ than the active controls (mean $=2.7$, range $=1.8-4.1)$, and the difference was statistically significant $(p=0.01)$. The MI group was also more likely to initiate treatment than the befriending group (96 vs. $80 \%$, again statistically significant, $p=0.02$ ). The main limitation of this simple study was its small sample. MI may be a good way to introduce adolescents to psychotherapy and get them more motivated. (This study was supported by the Capital and Coast District Health Board. The authors declared no conflict of interest.)

Dialectical behavior therapy (DBT) has been recognized as a fairly effective treatment for borderline personality disorder. The standard model of DBT for borderline personality disorder is a 12 -month, multimodal treatment, including individual sessions, skill groups, phone coaching and a therapist consultation team. As noted by McMain et al. [7], access to standard DBT is restricted due to limited resources and a shortage of well-trained therapists. Thus, they decided to test the effectiveness of a brief course of DBT skills training designed as an adjunctive intervention for high-risk suicidal patients with borderline personality disorder. This was a 2 -arm, singleblinded, prospective, randomized, 20-week trial comparing the effectiveness of a DBT skills training group with an active waiting list (WL) group (42 patients in each group). The DBT skills training consisted of a manualized approach developed by Linehan and adapted to a 20 -week curriculum in which groups met for $2 \mathrm{~h}$ weekly. The training used a psychoeducational approach to enhance

Clinical Factor 2016 capabilities. The following 5 modules were covered: mindfulness, emotion regulation, distress tolerance, interpersonal effectiveness and dialectics. Skills group leaders were not available to provide crisis coaching outside of group sessions. Patients were encouraged to have a therapist or another individual who could provide crisis support. Ancillary treatments were unrestricted in both groups. The primary outcome was the difference between DBT and WL in the frequency of suicidal and nonsuicidal self-injury behavior. The assessments of suicidal and nonsuicidal behavior were done at baseline, 10, 20, and 32 weeks. Forty-two subjects in the DBT group completed the treatment. There were no completed suicides in either the DBT or WL groups. The DBT group subjects showed greater reduction of suicidal and nonsuicidal selfinjurious behavior than the WL subjects at 32 weeks $(p<$ $0.0001)$. DBT subjects also showed greater improvement in measures of anger, distress tolerance, and emotion regulation than the WL subjects at week 32. Limitations of the study included the fact that there was no control over what the controls were doing while being on the WL (other treatments?), the self-report nature of the outcome measure, and the relatively short follow-up period. (The study was funded by the Ontario Mental Health Foundation. The costs of treatment were covered by the Canadian public health care system. The authors reported no conflict of interest.)

A significant proportion of patients with social anxiety disorder (SAD) respond only partially or fail to respond to antidepressants. Some studies suggested efficacy of the combination of pharmacotherapy and CBT (CBT for $\mathrm{SAD}$ ). However, no study examined the efficacy of combining antidepressants and CBT for patients who remain symptomatic despite an adequate trial with antidepressants. Thus, Yoshinaga et al. [8] conducted a prospective randomized open blinded end point 16-week single-center study comparing CBT combined with usual care (UC) compared to UC alone. UC included pharmacotherapy provided by a primary psychiatrist. The assessments by an independent assessor were done at baseline, week 8 , and week 16. Patients were evaluated by the Liebowitz Social Anxiety Scale (LSAS), the inclusion criterion was LSAS score $\geq 50$. Treatment response was defined as a $31 \%$ or greater reduction of the total LSAS score. Remission was defined as having a final LSAS score of $\leq 36$ and no longer meeting the DSM-IV-TR criteria for SAD. The CBT, conducted by 7 experienced therapists, consisted of 16 weekly sessions lasting $50 \mathrm{~min}$, though the protocol allowed up to six 90 -min sessions. There were 21 patients in each group. There was no significant difference be-

Psychother Psychosom 2017;86:323-331 DOI: $10.1159 / 000479820$ 
tween the groups in antidepressant (imipramine-equivalent) dose or anxiolytic (diazepam-equivalent) dose throughout the study. At the end of the study, the adjusted mean reduction in LSAS from the baseline was -40.87 for the CBT + UC group and +0.68 for the UC group (between-group difference $-41.55, p<0.0001$ ). The response rates were 85.7 and $10 \%(p<0.0001)$, and corresponding remission rates were 47.6 and $0 \%(p=0.0005)$ for CBT + UC versus UC alone. Significant differences were also found in favor of CBT + UC for social anxiety symptoms, depressive symptoms and functional impairment. The study was small and conducted in just one center; there was no psychological placebo-controlled condition and no follow-up data. Nevertheless, the results show that adding CBT to medication nonresponders or partial responders may be a reasonable strategy in treating SAD. (The study was supported by the Grant-in-Aid for the Japan Society for the Promotion of Science Fellows, a grant from the University of Miyazaki, a grant from the Japanese Ministry of Health, Labor and Welfare, and partly by the Special Budget for Project by the Japanese Ministry of Education, Culture, Sports, Science and Technology. The authors declared numerous honoraria from various pharmaceutical companies and other institutions.)

\section{Psychotherapy and Technology}

The evidence that mental health services, including psychotherapy, can be delivered over the Internet is growing. Thus, Kordy et al. [9] examined the efficacy of 2 Internet-delivered therapies/augmentation strategies in prolonging symptom-free intervals in patients with recurrent major depression after an acute phase of treatment. These 2 strategies were SUMMIT (Supportive Monitoring and Depression Management over the Internet) and SUMMIT-PERSON. SUMMIT is a fully automated Internet-delivered intervention, while SUMMITPERSON allows for consulting a clinical expert. Both strategies aim to prolong euthymia by signaling upcoming crises, assisting individuals in personal crisis management and facilitating early intervention if needed. An automated supportive monitoring system based on the Brief Patient Health Questionnaire PHQ-9 enabled patients to evaluate the course of their depression. An Internet discussion forum also provided peer support, and online provision for a crisis management plan allowed proactive coping with any upcoming crisis. The SUMMIT-PERSON contact with expert provided (a) monthly consultation group chats with a clinical expert and (b) one-on-one chat consultations with a clinical expert when the patient's monitoring signaled an upcoming crisis. Both interventions were compared with treatment as usual (TAU). TAU patients also received proactive preparation for recurrence of depressive symptoms through individual crisis management. Thus, this was a 12 -month, 3 -arm, multicenter, open-label, evaluator-blind, randomized controlled trial with 77 patients in SUMMIT, 79 in SUMMIT-PERSON, and 80 patients in TAU groups. All subjects received maintenance antidepressant medication and clinical management. The primary outcome measure was "well" and "unwell" weeks over 24 months, as determined by Psychiatric Status Rating of the Longitudinal Interval Follow-Up Evaluation (the evaluation was done every 6 months). SUMMIT compared to TAU reduced the time with an unwell status $(\mathrm{OR}=0.48$; $95 \% \mathrm{CI}=0.23$ $0.98)$ through faster transitions from unwell to well $(\mathrm{OR}=1.44 ; 95 \% \mathrm{CI}=0.83-2.50)$ and slower transitions from well to unwell $(\mathrm{OR}=0.69 ; 95 \% \mathrm{CI}=0.44-1.09)$. Contrary to the authors' hypothesis, SUMMIT-PERSON was not superior to either SUMMIT (OR $=0.77 ; 95 \%$ $\mathrm{CI}=0.38-1.56)$ or $\mathrm{TAU}(\mathrm{OR}=0.62 ; 95 \% \mathrm{CI}=0.31-1.24)$. For both interventions, the effect on transition from unwell to well was strongest after the intervention, i.e., at the 12-month evaluation, and weakened until the 24-month evaluation. No fatal outcomes occurred during the study. The data point to a preventive effect as well as to a crisis management effect. The authors made several points about the strengths of their study - their patients were severely ill, the course of their illness was rarely stable, and the attrition rates were low. They also suggested testing of an open-ended provision to increase the durability of the preventive effect of SUMMIT. There is no financial reason for a time limit, because SUMMIT is fully automated. Most costs are fixed, and variable costs emerge only throughout the introduction of the patient to the online system. (The study was funded by the German Research Foundation grant. Most authors had no conflict of interest. Dr. Hegerl had been on the advisory board of several pharmaceutical companies and speaker for several pharmaceutical companies. Dr. Rummer-Kluge received speaker honoraria from Janssen-Cilag. Dr. Vedder received honoraria for being on the advisory board of Otsuka Pharma.)

A small study by Ferwerda et al. [10] brought up a neglected issue in the area of Internet-based treatment - patient evaluation of the therapeutic relationship in Internet-based therapies. While some studies suggest that this relationship may be comparable to that of face-to-face treatments, Internet-based treatments may have incorpo- 
rated fewer features to develop and maintain a therapeutic relationship compared to face-to-face treatments. One of the issues has been that studies of the Internet-based interventions used instruments commonly used in the face-to-face interventions and not an instrument measuring specific aspects of the therapeutic relationship during Internet-based interventions. Thus, the authors developed the Internet-Specific Therapeutic Relationship Questionnaire (ITRQ) based on their review of the literature. The ITRQ has 9 items and 2 subscales, one called "Internet-specific time and attention" and the other termed "Internet-specific reflection and comfort." They compared this measure to the Working Alliance Inventory which is generally used for assessing the face-to-face treatment alliance. They administered both measures to 98 psoriasis and rheumatoid arthritis patients who participated in the treatment arm of 2 ongoing trials. Seventy-two pretreatment and 75 posttreatment questionnaires assessing the Internet-specific aspects of the treatment were available, and 52 patients completed both questionnaires. The internal consistence of the total scale was satisfactory (Cronbach's $\alpha=0.89$ ) as were the consistencies of both subscales (Cronbach's $\alpha=0.92$ and 0.87). The results also suggested that ITRQ is a possible predictor of general treatment satisfaction and patient-reported improvements after treatment. The authors felt that their results demonstrated the feasibility of the ITRQ as a new measure for Internet-specific aspects of the therapeutic relationship and support earlier findings for the therapeutic relationship as a possible predictor for treatment outcome. (This research was supported by grants from ZonMw, i.e., the Netherlands Organisation for Health Research and Development, and Pfizer. The authors had no conflict of interest.)

\section{Physical Consequences of Mental IIIness}

Hypertension is a major modifiable risk factor for cardiovascular disease in men and women. Exposure to trauma, including childhood abuse and combat, and posttraumatic stress disorder (PTSD), have been associated with an increased risk of hypertension. The understanding of the PTSD-hypertension in women is limited. Part of the problem has been the fact that most studies on PTSD and hypertension have been cross-sectional, and potential mediators have not been clearly identified. Sumner et al. [11] addressed the limitations of previous studies by examining associations between trauma exposure, PTSD symptoms, and risk of incident hypertension

Clinical Factor 2016 over 22 years in the Nurses' Health Study II (NHS-II), an ongoing longitudinal cohort study of younger and middle-aged women that began in 1989. They examined PTSD symptoms in response to a variety of traumas and incident hypertension. They also considered the effects of trauma and PTSD separately. The NHS-II includes 116,430 female nurses in the USA, aged $25-42$ years at enrollment in 1989. The women complete the questionnaires biennially, a follow-up is ongoing. In 2008, 60,804 women (aged 44-62) who completed the 2001 violence questionnaire and 2007 biennial questionnaire were mailed a supplementary questionnaire assessing trauma and PTSD. The response rate of returned questionnaires was $89 \%$ (54,224 women). Trauma exposure was measured with a 16-item modified version of the Brief Trauma Questionnaire, and the Short Screening Scale for DSM-IV PTSD was used to assess whether women ever experienced 7 PTSD symptoms in response to their worst trauma. The women reported lifetime physician-diagnosed hypertension at baseline in 1989. At each subsequent biennial questionnaire they indicated whether they had had physician-diagnosed hypertension in the past 2 years. Potential confounders included age at baseline, race, maximum parental education at the participant's birth, maternal and paternal history of hypertension, and age 5 somatotype (estimating childhood adiposity). Time-varying indicators included oral contraceptive use, menopausal status, hormone therapy use, current aspirin use, acetaminophen use, other nonsteroidal anti-inflammatory drug use, and physician-diagnosed hypercholesterolemia. Other variables examined included adult body mass index (BMI), smoking, alcohol consumption, physical activity, diet quality, sugar-sweetened and artificially sweetened beverage consumption, and lifetime antidepressant use. Many women (69\%) reported trauma at baseline. Of those, $73 \%$ reported no PTSD symptoms, $14 \%$ reported 1-3 symptoms, $8 \%$ reported $4-5$ symptoms, and 5\% reported 6-7 symptoms. Over the study, 5,144 additional women reported trauma exposure. Over the 22-year study period, 15,837 women developed hypertension. Time-updated PTSD symptoms were modestly associated with increased risk of hypertension in a dose-related fashion. Women with 6-7 PTSD symptoms had a $20 \%$ higher rate of developing hypertension than women without trauma, after adjusting for demographics, family history, and childhood adiposity. The findings were maintained, although attenuated after adjusting for hypertension-relevant medication, medical risk factors, and health behaviors. Only 2 potential mediators, BMI and antidepressant use, resulted in attenuation - BMI for

Psychother Psychosom 2017;86:323-331 DOI: $10.1159 / 000479820$ 
$30 \%$ and antidepressants for $21 \%$ - of the PTSD symptom-hypertension association. The data on antidepressants have to be viewed carefully, as higher PTSD symptoms were associated with a greater chance of antidepressant use. The study had a few limitations, such as the retrospective assessment of trauma and lifetime PTSD symptoms, and not addressing how remission of PTSD symptoms might impact the risk of hypertension. (The study was supported by NIMH grants and a Yerby Postdoctoral Fellowship. The authors had no conflict of interest.)

PTSD is not only associated with hypertension, but with other deleterious conditions, such as the metabolic syndrome (MetS). The direction of this association has not been properly established, as most studies of this association used cross-sectional designs. Wolf et al. [12] conducted a study to evaluate this relationship using a longitudinal design. They studied 1,355 subjects (655 men and 700 women) from the project VALOR (Veterans' After-Discharge Longitudinal Registry), a registry of VA mental health care users with or without PTSD who had been deployed to Iraq and Afghanistan. The subjects completed 2 waves of assessment approximately 2.5 years apart. Current (past month) PTSD was assessed via telephone using the PTSD module of the Structured Clinical Interview for DSM-IV (SCID) for the first evaluation and SCID for DSM-5 for the second evaluation. Data pertaining to MetS were extracted from the VA electronic medical records using laboratory values that were linked as closely as possible in time to the SCID-based PTSD assessment. The prevalence of MetS was 29\% among veterans with PTSD younger than 40 years at the time of the first assessment, which is significantly greater than the 20.3\% previously reported in an age-matched epidemiological sample. The prevalence of MetS among veterans without PTSD was $20.2 \%$. Cross-lagged panel models revealed that PTSD severity predicted subsequent increases in MetS severity $(p=0.002)$, after controlling for initial MetS severity, but MetS did not predict later PTSD symptoms. The analysis showed that for every additional PTSD symptom at the time of the first evaluation, the odds ratio for MetS diagnosis at the time of the second evaluation increased by $5.6 \%(p=0.003)$. The limitation of this study included the fact that metabolic profiles were not directly measured but extracted from medical records and that metabolic profiles of individuals prior to trauma exposure and PTSD onset were not available. PTSD seems to significantly contribute to the subsequent development of the MetS. (This study was supported by a Career Development Award to E.J.W. from the US Department of
Veterans Affairs, Clinical Sciences Research and Development Program, and grants from the Department of Defense, the NIMH, Department of Veterans Affairs, the National Institute on Aging and the National Center for Advancing Translational Sciences. The authors had no conflict of interest.)

Substance abuse is known to have deleterious effects on physical health. Methamphetamine, a widely abused and highly addictive substance, may cause widespread organ toxicity due to its central and peripheral sympathomimetic properties. As literature supporting the association between methamphetamine exposure and cardiovascular and cerebrovascular events mainly includes case reports, case series, postmortem studies and cross-sectional design-based research, Huang et al. [13] conducted a longitudinal cohort study with observation time ranging from 10 to 14 years by analyzing a nationwide Taiwanese population-based claims data set to assess the long-term cardiovascular and cerebrovascular complications among methamphetamine users. They retrospectively selected 1,315 inpatients treated for methamphetamine use between January 1, 1997, and December 31, 2000 (nested database), and matched them with a population proxy comparison group at a ratio of 1:4 through propensity score matching. All patients were monitored till December 31, 2010, for any incidence of cardiovascular disease or stroke, using the ICD-9-CM codes (primary outcome measure). Patients were mostly men (82.5\%) and approximately $50 \%$ of them were younger than 30 years. The total incidence of cardiovascular disease and stroke events significantly differed between methamphetamine users and controls $(87.5 / 10,000$ person-years in the methamphetamine group vs. $55.3 / 10,000$ personyears among controls, $p<0.001)$. The incidence of arrhythmia $(p=0.011)$ and hemorrhagic stroke $(p=0.001)$ was significantly higher in the methamphetamine group. The risk of these complications was also higher in the methamphetamine group. Age acted as an effect modifier for the risk estimation, the risk of cardiovascular sequelae was more significant in patients younger than 30 years ( $\mathrm{HR}=2.22, p=0.001)$, while the risk of stroke was higher among patients older than 30 years $(\mathrm{HR}=1.86, p=0.001)$. The study had several significant limitations. The ICD-9CM system does not have codes specific to methamphetamine use, and thus the best-estimate strategy for identifying subjects with methamphetamine use had to be developed. Methamphetamine users may have sought medical advice less often than controls. Some patients had probably been using methamphetamine long before being included in the study, which may lead to underes- 
timation of the effects of methamphetamine prior to the study. In some subjects cardiovascular events may not have been diagnosed accurately. Lastly, the authors did not use any statistical adjustment strategies such as Bonferroni correction. However, if a $p$ value $<0.005$ is reset as significant, all of the results except for arrhythmia remain the same. (This research was supported by grants from the National Science Council and Ministry of Science and Technology of Taiwan, and Taipei City Hospital, Taipei, Taiwan. The authors had no conflict of interest.)

\section{Novel Psychopharmacological Interventions}

As Jorge et al. [14] noted, prevention is more effective than treatment to decrease the burden of disease. It is important to know the cause or trigger of a disorder to be able to implement any preventive measure. Depressive disorders are a common event after traumatic brain injury (TBI). Several studies have reported the incidence of depressive disorder developing within a year following TBI being 29.5, 36.9, and even 53.1\%. TBI thus seems to be a good candidate for preventive measures, e.g., antidepressant medication, of its sequelae. Jorge et al. [14] examined the efficacy of pharmacological treatment to prevent the onset of depression following TBI. They enrolled 94 patients in a double-blind, placebo-controlled randomized controlled trial to examine the ability of sertraline to prevent TBI-associated depression. Patients between 18 and 85 years of age with a diagnosis of mild, moderate, or severe TBI who were required to have complete recovery of posttraumatic amnesia within 4 weeks of a traumatic episode were included in this 24 -week study. There were 48 patients ( 22 women) on sertraline $100 \mathrm{mg} /$ day and 46 patients (16 women) on placebo. Sertraline $25 \mathrm{mg} /$ day was given for 5 days, $50 \mathrm{mg} /$ day for the next 5 days and $100 \mathrm{mg} /$ day thereafter. Patients were evaluated in person using the Mini-International Neuropsychiatric Interview (MINI) at baseline and weeks 2, 4, 8, $12,16,20$, and 24 . In addition, the MINI was administered via telephone at weeks $6,10,14,18$, and 22 . The presence of mood disorders was ascertained by experienced psychiatrists who were unaware of treatment allocation. The primary outcome was the time to onset of a depressive disorder. The number of patients needed to treat to prevent the development of depression after TBI at 24 weeks was $5.9(95 \% \mathrm{CI}=3.1-71.1$; likelihood ratio test $\chi^{2}=4.6 ; p=0.03$ ) for sertraline versus placebo. All incident cases of depression had features of major depression. The influence of sertraline in the course of neuro- psychiatric variables was not detected. Sertraline was well tolerated. The limitations of this study included small sample size, 1 study center, predominance of mild and moderate TBI, and scarce representation of minorities (2\%). Nevertheless, this is the first trial indicating that antidepressants could be efficacious in preventing the onset of depression following TBI. (This study was funded by a grant from the NIMH. Dr. Jorge received honoraria from Xiang-Jansen Pharmaceuticals. Dr. Robinson reported compensation for participation in an advisory committee meeting by Avanir Pharmaceuticals, a lecture honorarium from Xiang-Jansen, research funding from the Senator Financial Group, royalties from Cambridge University Press, and serving as a consultant to Otsuka Pharmaceuticals. The remaining authors had no conflict of interest.)

The time delay of antidepressant action has been a major clinical problem, and thus several options to expedite it have been explored. It has been demonstrated that a single infusion of a low dose of ketamine rapidly improves depressive symptoms with efficacy onset within $1 \mathrm{~h}$ after infusion. However, this rapid and marked efficacy has been usually followed by a gradual loss of the therapeutic benefit, unless repeated infusions were implemented. Studies examining ketamine's role in the treatment of major depression (MDD) have several limitations, e.g., cross-over design, and targeting treatmentresistant depression, which limits the generalizability. Interestingly, the effects of add-on ketamine to antidepressant therapy have not been properly studied. Hu et al. [15] hypothesized that it is possible that a single dose of intravenous ketamine could speed up antidepressant efficacy and bridge the gap of the first weeks until clinically relevant antidepressant effects are seen with oral antidepressants. They thus decided to examine the antidepressant and antisuicidal effects and safety of a low-dose single intravenous ketamine infusion $(0.5 \mathrm{mg} / \mathrm{kg}$ over $40 \mathrm{~min})$ combined with escitalopram initiation in MDD. This was a randomized, double-blind, parallel-group placebo-controlled (for ketamine) trial of patients with nonpsychotic MDD. Thirty outpatients with severe MDD were randomized to 4 weeks of treatment with escitalopram 10 $\mathrm{mg} /$ day plus a single dose of intravenous ketamine (15 enrolled, 13 completed) or escitalopram $10 \mathrm{mg} /$ day plus placebo ( $0.9 \%$ i.v. saline; 15 enrolled, 14 completed). Depressive symptomatology was measured using the Montgomery-Asberg Depression Rating Scale (MADRS) and Quick Inventory of Depressive Symptomatology Self-Report (QIDS-SR). Suicidal ideation was evaluated using item 12 on the QIDS-SR. Other scales (Brief Psychiatric

Psychother Psychosom 2017;86:323-331 DOI: $10.1159 / 000479820$ 
Rating Scale, Young Mania Rating Scale, and ClinicianAdministered Dissociate States Scale) were used to assess adverse psychopathological effects. Patients were evaluated at baseline, 1, 2, 4, 24, and $72 \mathrm{~h}$ and 7, 14, 21, and 28 days. The primary outcome measure was time to response (response was defined as $\geq 50 \%$ MADRS score reduction). By 4 weeks, more escitalopram plus ketaminetreated patients than escitalopram plus placebo-treated patients responded ( 92.3 vs. $57.1 \%, p=0.04$ ) and remitted (76.9 vs. $14.3 \%, p=0.001$ ), with a significantly shorter time to response $(p<0.001)$ and remission $(p=0.01)$. The escitalopram plus ketamine group had also significantly lower MADRS and QIDS-SR scores from $2 \mathrm{~h}$ to 2 weeks, and QIDS-SR suicidality from 2 to $72 \mathrm{~h}$. The Young Mania Rating Scale scores increased significantly in the escitalopram plus ketamine group at 1 and $2 \mathrm{~h}$. It seems that a single dose of ketamine may speed up early oral antidepressant treatment response. (This study was funded by the National Science and Technology Major Projects for Major New Drugs Innovation and Development, and the Key Medical Specialties Development Project of Beijing Municipal Administration of Hospitals, People's Republic of China. Dr. Correll has been a consultant and/or advisor to or received honoraria from multiple pharmaceutical companies, including Lundbeck. The remaining authors reported no conflict of interest.)

Excoriation (skin-picking) disorder (SPD) is a disabling, underrecognized, yet fairly common (1.4-5.4\%) condition. As it has not been recognized as its own separate condition previously, its treatment has not been properly studied. Small studies of selective serotonin reuptake inhibitors produced mostly mixed effects. As glutaminergic dysfunction has been implicated in the pathophysiology of compulsive or habitual disorders such as SPD, Grant et al. [16] decided to study the role of $\mathrm{N}$-acetylcysteine in the treatment of SPD. N-acetylcysteine restores extracellular glutamate concentration in the nucleus accumbens which may therefore block reinstitution of compulsive behaviors. In addition, $\mathrm{N}$-acetylcysteine was found potentially useful in trichotillomania, a disorder linked to SPD. In this randomized, double-blind, placebo-controlled 12-week study, 66 patients were assigned either to $\mathrm{N}$-acetylcysteine (gradually increased from 1,200 to $3,000 \mathrm{mg} /$ day at week 6) or placebo. All patients met the DSM-5 criteria for excoriation disorder (some diagnosed were confirmed retrospectively as DSM-5 was published while this study was conducted). The study was conducted at 2 centers consecutively by the same principal investigator. Patients were evaluated by a number of scales (modified Yale-Brown Obsessive-Com- pulsive Scale [NE-YBOCS], Skin Picking Symptom Assessment Scale, Clinical Global Impression Improvement and Severity Scales; Sheehan Disability Scale, Hamilton Anxiety Rating Scale and Hamilton Depression Rating Scale, and Quality of Life Inventory). The primary outcome measure was the change from baseline using the YBOCS modified for Neurotic Excoriation (NR-YBOCS). Both NE-YBOCS subscales and other scales used were evaluated as secondary efficacy measures. The treatment with $\mathrm{N}$-acetylcysteine was associated with significant improvement. At baseline, NE-YBOCS scores were 18.9 and 17.9 for the treatment and placebo groups, respectively, and at week 12 the scores were 11.5 and 14.1 for the treatment and placebo groups, respectively $(p=0.048)$. The Clinical Global Impression Severity Scale baseline scores were 3.5 and 4.0 , and the 12 -week scores were 3.0 and 4.2 , respectively $(p=0.003)$. At the end of the study, $15 / 32$ patients (47\%) receiving $\mathrm{N}$-acetylcysteine and 4/1 (19\%) patients receiving placebo were much or very much improved $(p=0.03)$. $\mathrm{N}$-acetylcysteine was well tolerated. (Great American Health provided the $\mathrm{N}$-acetylcysteine to the investigational pharmacies and provided certificates of analysis and conformance to ensure compliance with the standards of Good Manufacturing Practice. Great American Health had no role in this study and manuscript preparation. Drs. Grant, Chamberlain, and Odlaug reported honoraria and support from various pharmaceutical companies and publishing houses. Dr. Odlaug is currently [after the study] employed by H. Lunbeck A/S. The remaining authors reported no conflict of interest.)

\section{Disclosure Statement}

The author declares no conflict of interest.

References

1 Fava GA: The clinical factor. Psychother Psychosom 2011;80:1-3.

2 Balon R: Clinical factor 2010. Psychother Psychosom 2011;80:195-198.

3 Niemela S, Sourander A, Surcel H-M, Hinkka-Yli-Salomaki S, McKeague IW, CheslackPostava K, Brown AS: Prenatal nicotine exposure and risk of schizophrenia among offspring in a national birth cohort. Am J Psychiatry 2016;173:799-806.

4 Laursen TM, McGrath JJ: The strange case of smoking and schizophrenia - the epidemiology detectives are on the trail. Am J Psychiatry 2016;173:757-758.
Balon 
5 Hodgins S, Larm P, Westerman J: Individuals developing schizophrenia are hidden among adolescent substance misusers. Psychol Med 2016;46:3041-3050.

6 Dean S, Britt E, Bell E, Stanley J, Collings S: Motivational interviewing to enhance adolescent mental health treatment engagement: a randomized clinical trial. Psychol Med 2016; 46:1961-1969.

7 McMain SF, Guimond T, Barnhart R, Habinski L, Streiner DL: A randomized trial of brief dialectical behavior therapy skills training in suicidal patients suffering from borderline disorder. Acta Psychiatr Scand 2016;135:138148.

8 Yoshinaga N, Matsuki S, Niitsu T, Sato Y, Tanaka M, Ibuki H, Takanashi R, Ohshiro K, Ohshima F, Asano K, Kobori O, Yoshimura K, Hirano Y, Sawaguchi K, Koshizaka M, Hanaoka $H$, Nakagawa A, Nakazato M, Iyo M, Shimizu E: Cognitive behavioral therapy for patients with social anxiety disorder who remain symptomatic following antidepressant treatment: a randomized, assessor-blinded, controlled trial. Psychother Psychosom 2016;85:208-217.
9 Kordy H, Wolf M, Aulich K, Burgy M, Hegerl U, Husing J, Puschner B, Rummel-Kluge C, Vedder $\mathrm{H}$, Backenstrass $\mathrm{M}$ : Internet-delivered disease management for recurrent depression: a multicenter randomized controlled trial. Psychother Psychosom 2016;85: 91-98.

10 Ferwerda M, van Beugen S, van Rief PCLM, van der Kerkhof PCM, de Jong EMGJ, Smit JV, Zeeuwen-Franssen MEJ, Kroft EBM, Visser H, Vonkeman VE, Creemers MCW, van Middendorp H, Evers AWM: Measuring the therapeutic relationship in Internedbased interventions. Psychother Psychosom 2016;85:47-49.

11 Sumner JA, Kubzansky LD, Roberts AL, Gilsanz P, Chen Q, Winning A, Forman JP, Rimm EB, Koenen KC: Post-traumatic stress disorder symptoms and risk of hypertension over 22 years in a large cohort of younger and middle-aged women. Psychol Med 2016;46: 3105-3116.

12 Wolf EJ, Bovin MJ, Green JD, Mitchell KS, Stoop TB, Baretto KM, Jackson CE, Lee LO, Fanf SC, Trachtenberg F, Rosen RC, Keane TM, Marx PB: Longitudinal associations between post-traumatic stress disorder and metabolic syndrome severity. Psychol Med 2016;46:2215-2226.
13 Huang M-C, Yang S-Y, Lin S-K, Chen K-Y, Chen Y-Y, Kuo C-J, Hung Y-N: Risk of cardiovascular disease and stroke events in methamphetamine users: a 10-year follow-up study. J Clin Psychiatry 2016;77:1396140316.

14 Jorge R, Acion L, Burin DI, Robinson RG: Sertraline for preventing mood disorder following traumatic brain injury. A randomized clinical trial. JAMA Psychiatry 2016;73:10411047.

15 Hu Y-D, Xiang Y-T, Fang J-X, Zu S, Sha S, Shi H, Ungvari GS, Correll CU, Chiu HFK, Xue Y, Tian TF, Wu A-S, Ma X, Wang G: Single i.v. ketamine augmentation of newly initiated escitalopram for major depression: results from a randomized, placebo-controlled 4-week study. Psychol Med 2016;43:626-635.

16 Grant JE, Chamberlain SR, Redden SA, Leppink EW, Odlaug BL, Kim SW: N-acetylcysteine in the treatment of excoriation disorder. A randomized clinical trial. JAMA Psychiatry 2016;73:490-496. 